\title{
Switching Host Metabolism as an Approach to Dampen SARS-CoV-2 Infection
}

\author{
Sameh Soliman ${ }^{\mathrm{a}}$ MoezAllslam E. Faris ${ }^{\mathrm{b}}$ Zakaria Ratemi ${ }^{\mathrm{c}} \quad$ Rabih Halwani $^{\mathrm{d}}$ \\ aDepartment of Medicinal Chemistry, College of Pharmacy, University of Sharjah, Sharjah, UAE; ${ }^{b}$ Department \\ of Clinical Nutrition and Dietetics, College of Health Sciences, University of Sharjah, Sharjah, UAE; 'Faculté de \\ Médecine, Université de Montréal, Montréal, QC, Canada; ${ }^{\mathrm{d} D e p a r t m e n t}$ of Clinical Sciences, College of Medicine, \\ University of Sharjah, Sharjah, UAE
}

\section{Keywords}

Nutrition · Metabolic switch · COVID-19 · Ketogenic diet · Intermittent fasting $\cdot$ Medium-chain triglycerides

\begin{abstract}
Background: COVID-19 pandemic, a global threat, adversely affects all daily lives, altered governmental plans around the world, and urges the development of therapeutics and prophylactics to avoid the expansion of the viral infection. With the recent gradual opening after long lockdown, several recommendations have been placed, with dietary modification as one of the most important approaches that have been appraised. Summary: Here, we are reviewing how changing the host metabolism, particularly changing the host metabolic state from the carbohydrate-dependent glycolytic state to a fat-dependent ketogenic state, may affect viral replication. Furthermore, the impact of intermittent fasting (IF) in triggering metabolic switch along with the impact of supplementation with medium-chain triglycerides (MCTs) such as lauric acid in repressing the envelope formation and viral replication is also addressed. The amalgamation of IF and a ketogenic diet rich in MCTs is thought to work as a prophylactic measure for normal people and adjunct therapy for
\end{abstract}

karger@karger.com www.karger.com/anm

(c) 2020 S. Karger AG, Base

Karger" infected persons. Key Message: A diet regimen of ketogenic breakfast along with supplementation with two doses of lauric acid-rich MCTs at breakfast and lunch times, followed by 8-12-h IF and a dinner rich with fruits and vegetables, could be a potential prophylactic strategy and adjuvant therapy to combat SARS-CoV-2 infections.

(c) 2020 S. Karger AG, Basel

\section{Introduction}

The outbreak of the current global pandemic caused by the spread of a novel severe acute respiratory syndrome coronavirus-2 (SARS-CoV-2; COVID-19) has posed an unprecedented threat to global health and the world economy. COVID-19 has shaken up all daily lives, and governments around the world are encouraging precautionary measures to help stem the spread of infections. With the recent gradual opening after long lockdown, several recommendations have been suggested. Among these, dietary modification is one of the most important approaches that have been discussed to strengthen human immunity against the spread of viral infection [1-4]. However, to propose a special diet during this latent 
phase of viral spread, it is mandatory to carefully understand the viral-host metabolism relationship.

In general, viruses are parasites that lack a defined metabolism [5], thus rely on the host cell metabolism for the acquisition of raw materials and energy necessary for their biological processes $[2,5]$. Viral infections alter the host metabolism including, in particular, glycolysis and fatty acid synthesis [6] to initiate an environment required for the replication and spread of the virus in the human body. Alteration of host cell metabolism by viruses is vital to provide specific substrates that are uniquely needed at high levels for virion production and assembly. Virus benefits from the alteration of host metabolism by accessing (i) the host nucleotides for viral genome replication, (ii) amino acids for virion assembly, (iii) carbohydrates as an energy source, and (iv) fatty acids for viral envelope formation and glycoprotein involvement. In fact, the metabolic status of the virus-contracted hosts, their dietary intakes, sex, age, lifestyle, and environmental factors they are exposed to, as well as their medical conditions, all determine the prognosis of the cases and the clinical severity of SARS-CoV-2 infections [2].

Initial preliminary data, using the ecological observational study designed at the global level, indicated an inverse relationship between the number of cases/deaths from COVID-19 and the total intakes of fruits and vegetables (Faris et al. 2020, unpublished data). These preliminary findings suggest a role for the dietary intakes and nutritional status in favoring or disfavoring the body environment required for viral growth and replication and signify the importance of dietary factors in the battle against the pandemic infection [4].

Interestingly, dietary modifications have been proposed, including fasting and caloric restrictions, as one of the approaches to alter metabolic pathways [7]. Intermittent fasting (IF) is an ancient practice followed by different populations globally and has different formats, including time-restricted feeding, alternate-day fasting, 5:2 IF (fasting 2 days, each week), and religious forms of IF $[8,9]$ such as Ramadan IF [10]. During IF, the fasting subjects alternate between periods of voluntary abstinence from food and drink. As a result of this alternation in feeding and fasting periods, metabolic alterations consequently occur to cope with the changes in energy supply and energy shortage, respectively. Halting intakes of dietary long-chain fatty acids (LCFA) and supplementation with medium-chain triglycerides (MCTs) can result in negative energy balance and weight loss by increasing host energy expenditure and lipid oxidation, which disfavor the microenvironment of viral replication [11].
Metabolic switching by performing special eating and exercise pattern is a well-established strategy that affects the host metabolism and can lead to improving healthrelated conditions [12]. Here, we are proposing that designing a dietary regime can switch the host metabolism to inhibit viral replication and invasion. This is a very safe therapeutic and prophylactic approach that can easily be followed by a wide range of individuals with variable comorbidities [13].

\section{Importance of Host Lipids to SARS-CoV-2 Infections}

It is well understood that fatty acid synthesis is integral for the creation of lipid material in the cell and is vital for increased membrane production as well as other cellular needs [14]. Importantly, many viruses induce and require fatty acid synthesis for their life cycle [15]. Throughout this cycle, lipids play essential roles as they mediate the fusion of viral envelopes with host cell membranes, contribute to the formation of the viral replication complex, and generate the energy needed for viral replication [16]. Inhibition of saturated LCFA synthesis indicates that fatty acid is required for the production of infectious virions [14]. It appears that fatty acid synthesis is utilized to build the virion envelope [17], whereas LCFA were found at high levels in the virion envelope [17]. It is more likely that these LFCFA are paramount for the integrity of the virion envelope and binding to host cells [17]. Furthermore, it has been reported that fatty acid synthesis is needed to increase the glycoprotein levels of some viruses such as varicella-zoster virus [18].

The question now is whether SARS-CoV-2 similarly affects the host lipid metabolism. A better understanding of SARS-CoV-2-induced metabolic alterations may lead to the design of a special dietary approach that can switch the metabolic environment to disfavor the existence of the virus in the human body. Targeting the host metabolic pathway can provide a safer prevention approach that can overcome the challenges of a virus targeting drugs, such as the development of drug resistance.

To better understand how SARS-CoV-2 can alter the host metabolism, it is important to identify the main features of the virus and its closest match, since no data regarding the SARS-CoV-2-host metabolic changes are available yet. Coronaviruses, including SARS-CoV-2, are enveloped viruses with a nucleocapsid mainly made of phosphorylated nucleocapsid protein embedded inside a phospholipid bilayer envelop. This envelope plays an important role in virus assembly and release, and it is critical for viral pathogenesis $[19,20]$. The viral surface consists of the spike protein of the virus, which is essential for the 
attachment to the host cell receptor and for initiating infection $[21,22]$.

Although no data are describing how the host lipid metabolism affects SARS-CoV-2 infection, it has been shown that glycerophospholipids and fatty acids are significantly elevated in coronavirus-infected cells. Besides, coronaviruses attack the host cell intracellular membranes to create specific phospholipids to form replicative organelles suitable for virus genome replication [23].

\section{Switching the Host Lipid Metabolism Can Stop Virus \\ Replication and Assembly}

A change in the metabolic pathway is called "metabolic switching" [24]. Among these metabolic alterations, the most significant metabolic change is the shift from the glucogenic/glycolytic pathway (fed state) to the ketogenic pathway in the liver (fast state) [13]. Switching body metabolism from fed/feasting state to a fasting state involves systemic and cellular remarkable adaptations to the bioenergetic challenges (from glucose to ketogenesis). This can cause an increase in ketones (acetoacetate and $\beta-\mathrm{HB}$ ), increase in mitochondrial stress resistance, increase antioxidant defenses, increase autophagy, increase DNA repair, decrease insulin secretion, decrease mTOR, and decrease protein synthesis [13]. In the absence of current treatment for SARS-CoV-2, a strong interest has emerged regarding the optimization of the intake of dietary factors, specifically lipids, to effectively reduce inflammation and oxidative stress, thus strengthening the immune response during the COVID-19 crisis. Besides, metabolic changes accompanied by IF that trigger antiviral mechanism has been suggested elsewhere $[25,26]$.

Given the indispensable nature of lipids in multiple stages of viral replication, the modulation of lipid profiles in host cells has been recently proposed as a potential novel strategy to combat human coronaviruses [16]. Bioactive lipids with antiviral properties cited in the literature include certain long-chain monounsaturated fatty acids (MUFAs) and medium-chain fatty acids (MCFAs), specifically lauric acid (LA). LA, the primary fatty acid of coconut oil, is a saturated MCFA with potent antimicrobial properties [27]. When ingested, coconut oil is metabolized to produce the metabolite monolaurin [28].

Medium-chain saturated fatty acids such as LA and long-chain unsaturated fatty acids (such as oleic acid of olive oil) are highly active against enveloped viruses such as coronavirus $[29,30]$. Monoglycerides of these fatty acids also show significant antiviral activity. Antiviral fatty acids impact the viral envelope, causing leakage and at higher concentrations can cause a complete disintegration of the viral particles including the viral envelope [29].

The antiviral effect of MCFAs including LA has been tested against arenavirus infection; LA showed the most active inhibitory effect against viral replication since it caused a significant decrement in the virus yields of several pathogenic strains in a dose-dependent manner, without affecting the cell viability [31]. Furthermore, LA was found to inhibit a late maturation stage in the replicative cycle of the Junin virus (JUNV). Viral protein synthesis was not affected by the compound, but the expression of glycoproteins in the plasma membrane was diminished. A direct correlation between the inhibition of JUNV production and stimulation of triacylglycerol cell content was demonstrated. On the other hand, shorteror longer-chain fatty acids showed a reduced or negligible anti-multiplication effect on the JUNV [31]. Both triacylglycerol- and LA-induced effects were dependent on the continued presence of fatty acid. Thus, the reduced insertion of viral glycoproteins into the plasma membrane, apparently due to the increased incorporation of triacylglycerols, seems to inhibit JUNV maturation and release [31].

The antiviral activity of LA and monolaurin can be achieved through 3 distinct mechanisms. First, LA and monolaurin inactivate viruses by disintegrating the viral envelope, thus reducing the infectivity of RNA and DNA enveloped viruses [32]. Second, LA inhibits the late maturation stage in the virus replication cycle as demonstrated in a study investigating the LA-mediated inhibition of the Junin virus, which possesses a similar structure to SARS-CoV-2 $[33,34]$. Third, LA prevents the binding of viral proteins to the host cell membrane as described for infectious Vesicular stomatitis virus[35]. The effectiveness and safety of LA as an antiviral compound against HIV were also suggested $[36,37]$. The substantial evidence for the antiviral properties of LA (commonly found in coconut oil) is promising and encourages its further investigation as a potential therapeutic agent that is both affordable and clinically innocuous for SARS-CoV-2-infected patients.

Recent research signified the importance of MCFA supplementation (in the form of C8:0-C10:0 MCT supplement) as a way to optimize ketogenicity induced upon metabolic switching [38]. An 8-h metabolic study protocol was suggested to assess the influence of ketogenic MCT supplement, meal timing, and low-carbohydrate meal on plasma levels of free fatty acids (FFA), blood ketones, glucose, and insulin. Over the 8 -h test period, the provision of the study participants with 2 doses of the ketogenic MCT drink (10-g MCT for each dose of the drink) 
increased the plasma ketone response by $88 \%$ while reducing glycemic response by $12 \%$, without changing insulin or FFA levels. Besides, researchers found that subsequent to an overnight fast, an early breakfast was more effective in potentiating the ketogenic effect of metabolic switch over $8 \mathrm{~h}$ in comparison with a delayed lunch without breakfast. Researchers concluded that a ketogenic MCT drink can provide a booming short-term increase in plasma levels of ketones under various feeding conditions and might augment the metabolic effects of a shortterm metabolic switch or IF [38].

\section{Switching Lipid Metabolism and Inhibition of Cytokine Storm}

Once SARS-CoV-2 invades the cells, it hijacks the cells' machinery and uses it to ensure its proper replication. This disruption results in damaging the infected cells [39]. This prompts a local immune response, involving the recruitment of macrophages and monocytes that often readily subsides permitting patients to ultimate recovery [39]. However, in more severe cases, viral replication triggers an aberrantly strong release of proinflammatory cytokines, a phenomenon termed as a cytokine storm, which causes damage to host tissue and contributes to lung injury and respiratory failure [39].

The positive effect of IF in triggering autophagy, and the way how autophagy triggers the innate and adaptive immunity against viral infection, including the SARS$\mathrm{CoV}-2$, is extensively reviewed [40]. Hannan and colleagues [26] reported that fasting can provoke the host defense system through activating multiple physiological processes, including autophagy and immune responses. The pulmonary alveolar epithelial cells that are infected with SARS-CoV-2 release damage-associated molecular patterns such as nucleic acids, which are recognized by adjacent epithelial cells and resident macrophages, prompting the release of proinflammatory cytokines and chemokines (IL-6, IP-10, MIP1 $\alpha$, and MCP1). These mediators attract inflammatory cells, including monocytes, macrophages, and $\mathrm{T}$ cells to the site of infection, which normally acts on resolving the viral infection. In case of a dysfunctional immune response, the uncontrolled viral replications, as well as the massive infiltration of inflammatory cells, destroy the airway epithelial cells, resulting in the release of several proinflammatory mediators including IL- $1 \beta$, IL- 6 , and TNF- $\alpha$. Another fasting-mediated cellular process is autophagy that either activates innate and adaptive immunity or degrades viral particles (xenophagy) [26].
Besides its direct antiviral effect, MCTs were reported to enhance macrophage phagocytic activity and regulate the expression of several inflammatory mediators [41]. MCFAs including caprylic, capric, and LAs were also shown to suppress the expression of IL-8 [41], a proinflammatory cytokine, and the main chemoattractantdriving neutrophil lung infiltration.

During SARS-CoV-2 infection, the uncontrolled viral replication in lung tissue triggers the release of a wide range of proinflammatory cytokines including IL- $1 \beta$, IL6 , IL-8, and TNF- $\alpha$ [39]. Three of the aforementioned cytokines have been reported to be significantly reduced upon IF, which reinforces the hypothesized role of IF in ameliorating the inflammatory background of the infection $[42,43]$. The infiltration of neutrophils to the airway tissue was widely reported especially in severe stages of the disease. These neutrophils, while attacking infected cells, contribute largely to the destruction of the lung tissue and hence the development of acute respiratory distress syndrome (ARDS). Therefore, by regulating IL- 8 expression, MCFAs may limit neutrophil infiltration and hence help to protect lung tissue from the exaggerated immune response.

Lauric acid, on the other hand, was shown to downregulate the expression of IL-6 in intestinal epithelial cells [41]. Interleukin-6 is another key proinflammatory cytokine contributing to the cytokine storm observed in most patients with severe COVID-19 symptoms. It is released from macrophages, and dendritic cells are infected with SARS-CoV-2 [44]. Further, IL-6 triggers the release of vascular endothelial growth factor, CCL2, and IL-8 [45], causing an upregulation of C-reactive protein blood levels and increased infiltration of macrophages and neutrophils to the inflamed lung tissue. The cytokine suppressive effect of LA suggests that it may help in preventing or at least regulating the uncontrolled wave of proinflammatory cytokine and its devastating systemic effect.

\section{Suggested Diet to Disfavor the Viral Replication and} Infection

Here, we propose a medical hypothesis either as a prophylactic or adjuvant supplement for people infected with SARS-CoV-2. This aims to mitigate the infection by changing the microenvironment of the host metabolism in a way that disfavors viral replication and controls the aggressive nonspecific immune responses. This is based on a metabolic switch accompanied by host lipid metabolism to the ketogenic pathway. Caprylic acid/LA-enriched coconut oil can be used as a ketogenic MCT sup- 
Fig. 1. Schematic representation of a diet regimen proposed to dampen the SARSCoV-2 infection by triggering metabolic switching. SARS-CoV-2, severe acute respiratory syndrome coronavirus-2; MCFAs, medium-chain fatty acids; IF, intermittent fasting.

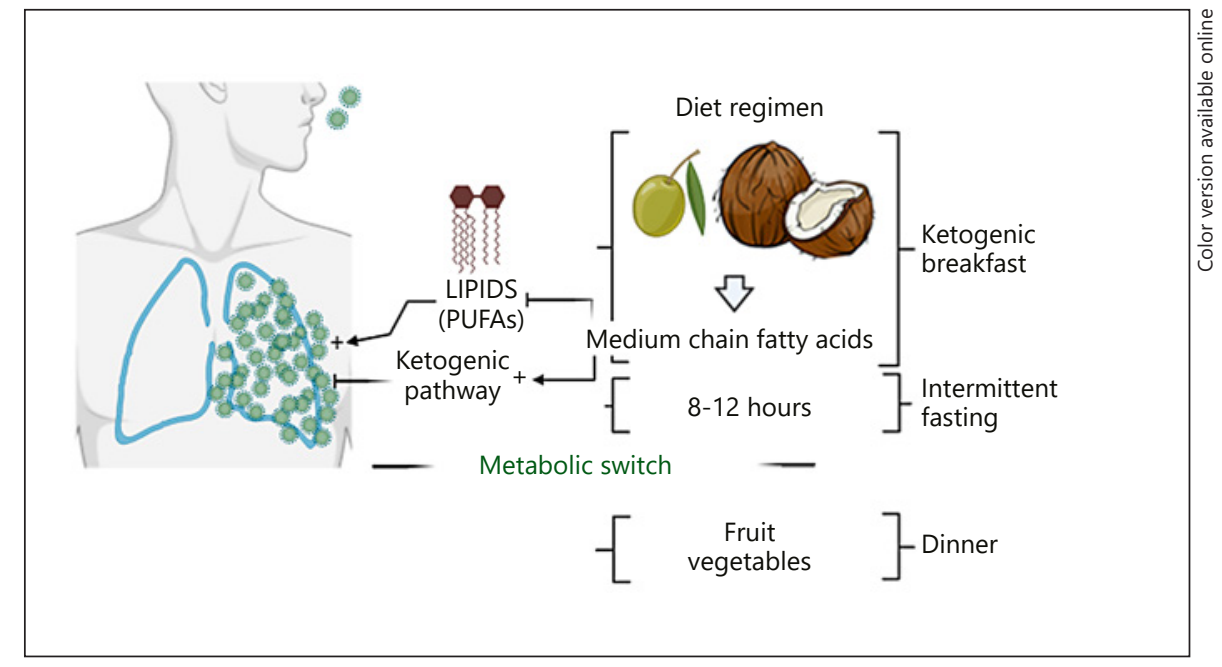

plement that is hypothesized to aid in suppressing viral replication and ameliorating the severity of COVID-19 complication. Also, by enhancing macrophage activity, limiting neutrophil infiltration into the lung tissue, as well as suppressing IL-6 cytokine, one of the key players in the cytokine storm observed at later severe stages of the diseases, MCFAs may suppress uncontrolled immune responses.

This modification of the microenvironment required for viral replication can be accomplished as well by a ketogenic MCT drink rich in LA and low in LCFAs followed by a short-term IF period of 8-12 h. For a more practical medical nutrition protocol, here we suggest that healthy individuals, or patients with COVID-19, are suggested to follow the proposed dietary regime indicated below and in Figure 1, as a prophylactic or therapeutic approach:

1. A breakfast enriched with ketogenic MCT via coconut oil enriched with LA/caprylic acid and monounsaturated long-chain fatty acid (oleic acid 18:1 omega-9) from olive oil rich in antioxidant and anti-inflammatory bioactive phytochemicals [46]. Breakfast with more protein foods prepared with MCT oil (coconut oil) can trigger the ketogenic pathway and hence supposed to repress the viral replication.

2. Following breakfast supplemented with 20 -g ketogenic MCT drink and keeping an 8-12 h fasting can trigger the ketogenic pathway and allow for cellular activation of the autophagy pathway and hence proposed to ameliorate the SARS-CoV-2 viral replication. At lunch time, subjects are given 20-g ketogenic MCT drink without a meal.

Metabolic Switch and SARS-CoV-2 Infection
3. After the $8-12$ fasting period, a dinner with a balanced diet can provide the required amount of carbohydrates, and fibers from fruits and vegetables rich in vitamins and minerals are important for boosting the immunity against SARS-CoV-2 infection [1].

\section{Conclusion}

With the gradual opening after long lockdown because of the COVID-19 pandemic, we are proposing a prophylactic diet regimen that may limit viral levels in the human body. This can be established by switching the host lipid metabolism, critical for viral replication and assembly, by induction of the ketogenic pathway using coconut-rich MCFAs along with olive oil, followed by $8-12 \mathrm{~h}$ fasting and a dinner rich with fruits and vegetables.

\section{Acknowledgment}

The authors acknowledge the generous support from Sandooq Al Watan and University of Sharjah to Sameh Soliman.

\section{Conflict of Interest Statement}

The authors confirm that this article content has no conflicts of interest. 


\section{Funding Sources}

The current work did not receive any grant or fund.

\section{Author Contributions}

MoezAlIslam Faris and Sameh Soliman: conception and design of the work; Rabih Halwani: interpretation of findings of the work; MoezAlIslam Faris, Sameh Soliman, and Zakaria Ratemi: drafting the work; Rabih Halwani: revising it critically for important intellectual content. All authors approved the final version to be published.

\section{References}

1 Calder PC. Nutrition, immunity and COVID-19. BMJ Nutr Prev Health. 2020;3(1):7492.

2 Gasmi A, Noor S, Tippairote T, Dadar M, Menzel A, Bjørklund G. Individual risk management strategy and potential therapeutic options for the COVID-19 pandemic. Clin Immunol. 2020;215:108409.

3 Iddir M, Brito A, Dingeo G, Fernandez Del Campo SS, Samouda H, La Frano MR, et al. Strengthening the immune system and reducing inflammation and oxidative stress through diet and nutrition: considerations during the COVID-19 crisis. Nutrients. 2020;12(6): 1562.

4 Zabetakis I, Lordan R, Norton C, Tsoupras A. COVID-19: the inflammation link and the role of nutrition in potential mitigation. Nutrients. 2020;12(5):1466.

5 Villarreal L. Are viruses alive? Sci Am. 2005 Jan 01;291:100-5.

6 Sanchez EL, Lagunoff M. Viral activation of cellular metabolism. Virology. 2015 May 01; 479-80:609-18.

7 Burns J, Manda G. Metabolic pathways of the Warburg effect in health and disease: perspectives of choice, chain or chance. Int J Mol Sci. 2017;18(12):2755.

8 Patterson RE, Sears DD. Metabolic effects of intermittent fasting. Annu Rev Nutr. 2017; 37(1):371-93.

9 Di Francesco A, Di Germanio C, Bernier M, de Cabo R. A time to fast. Science. 2018; 362(6416):770-5.

10 Faris MAIE, Jahrami HA, Alsibai J, Obaideen AA. Impact of Ramadan diurnal intermittent fasting on the metabolic syndrome components in healthy, non-athletic Muslim people aged over 15 years: a systematic review and meta-analysis. Br J Nutr. 2020;123(1):1-22.

11 Mumme K, Stonehouse W. Effects of medium-chain triglycerides on weight loss and body composition: a meta-analysis of randomized controlled trials. J Acad Nutr Diet. 2015;115(2):249-63.

12 Mattson MP, Moehl K, Ghena N, Schmaedick $\mathrm{M}$, Cheng A. Intermittent metabolic switching, neuroplasticity and brain health. Nat Rev Neurosci. 2018 Feb 01;19(2):63-80.

13 De Cabo R, Mattson MP. Effects of intermittent fasting on health, aging, and disease. N Engl J Med. 2019;381(26):2541-51.
14 Munger J, Bennett BD, Parikh A, Feng XJ, McArdle J, Rabitz HA, et al. Systems-level metabolic flux profiling identifies fatty acid synthesis as a target for antiviral therapy. Nat Biotechnol. 2008 Oct 01;26(10):1179-86.

15 Herker E, Ott M. Unique ties between hepatitis $C$ virus replication and intracellular lipids. Trends Endocrinol Metab. 2011 Jun 01;22(6): 241-8.

16 Yan B, Chu H, Yang D, Sze KH, Lai PM, Yuan $\mathrm{S}$, et al. Characterization of the lipidomic profile of human coronavirus-infected cells: implications for lipid metabolism remodeling upon coronavirus replication. Viruses. 2019 Jan 16;11(1):73.

17 Koyuncu E, Purdy JG, Rabinowitz JD, Shenk T. Saturated very long chain fatty acids are required for the production of infectious human cytomegalovirus progeny. PLoS Pathog. 2013;9(5):e1003333.

18 Namazue J, Kato T, Okuno T, Shiraki K, Yamanishi K. Evidence for attachment of fatty acid to Varicella-zoster virus glycoproteins and effect of cerulenin on the maturation of Varicella-zoster virus glycoproteins. Intervirology. 1989;30(5):268-77.

19 Lundin A, Dijkman R, Bergström T, Kann N, Adamiak B, Hannoun C, et al. Targeting membrane-bound viral RNA synthesis reveals potent inhibition of diverse coronaviruses including the Middle East respiratory syndrome virus. PLoS Pathog. 2014 May; 10(5):e1004166.

20 Johnson BA, Graham RL, Menachery VD. Viral metagenomics, protein structure, and reverse genetics: key strategies for investigating coronaviruses. Virology. 2018 Apr;517:30-7.

21 Ou X, Liu Y, Lei X, Li P, Mi D, Ren L, et al. Characterization of spike glycoprotein of SARS-CoV-2 on virus entry and its immune cross-reactivity with SARS-CoV. Nat Commun. 2020 Mar 27;11(1):1620.

22 Walls AC, Park YJ, Tortorici MA, Wall A, McGuire AT, Veesler D. Structure, function, and antigenicity of the SARS-CoV-2 spike glycoprotein. Cell. 2020 Apr;181(2):281-92. e3.

$23 \mathrm{Xu} \mathrm{K}$, Nagy PD. RNA virus replication depends on enrichment of phosphatidylethanolamine at replication sites in subcellular membranes. Proc Natl Acad Sci U S A. 2015; 112(14):E1782-91.
24 Hernandez A, Truckenbrod L, Federico Q, Campos K, Moon B, Ferekides N, et al. Metabolic switching is impaired by aging and facilitated by ketosis independent of glycogen. Aging. 2020;12(9):7963-84.

25 Faris MAIE, Salem ML, Jahrami HA, Madkour MI, BaHammam AS. Ramadan intermittent fasting and immunity: an important topic in the era of COVID-19. Ann Thorac Med. 2020;15(3):125-133.

26 Hannan MA, Rahman MA, Rahman MS, Sohag AAM, Dash R, Hossain KS, et al. Intermittent fasting, a possible priming tool for host defense against SARS-CoV-2 infection: crosstalk among calorie restriction, autophagy and immune response. Immunol Lett. 2020 Oct 01;226:38-45.

27 Lappano R, Sebastiani A, Cirillo F, Rigiracciolo DC, Galli GR, Curcio R, et al. The lauric acid-activated signaling prompts apoptosis in cancer cells. Cell Death Discov. 2017;3:17063.

28 McCarty MF, DiNicolantonio JJ. Lauric acidrich medium-chain triglycerides can substitute for other oils in cooking applications and may have limited pathogenicity. Open Heart. 2016;3(2):e000467.

29 Thormar H, Isaacs CE, Brown HR, Barshatzky MR, Pessolano T. Inactivation of enveloped viruses and killing of cells by fatty acids and monoglycerides. Antimicrob Agents Chemother. 1987;31(1):27-31.

30 Boateng L, Ansong R, Owusu W, SteinerAsiedu M. Coconut oil and palm oil's role in nutrition, health and national development: a review. Ghana Med J. 2016;50(3):189-96.

31 Bartolotta S, García CC, Candurra NA, Damonte EB. Effect of fatty acids on arenavirus replication: inhibition of virus production by lauric acid. Arch Virol. 2001;146(4):777-90.

32 Hierholzer JC, Kabara JJ. In vitro effects of monolaurin compounds on enveloped RNA and DNA viruses. J Food Saf. 1982 Mar;4(1): $1-12$.

33 Bartolotta S, García CC, Candurra NA, Damonte EB. Effect of fatty acids on arenavirus replication: inhibition of virus production by lauric acid. Arch Virol. 2001;146(4):777-90.

34 Grant A, Seregin A, Huang C, Kolokoltsova O, Brasier A, Peters C, et al. Junín virus pathogenesis and virus replication. Viruses. 2012 Oct 19;4(10):2317-39. 
35 Hornung B, Amtmann E, Sauer G. Lauric acid inhibits the maturation of vesicular stomatitis virus. J Gen Virol. 1994 Feb;75(Pt 2):353-61.

36 Hardon A, Desclaux A, Egrot M, Simon E, Micollier E, Kyakuwa M. Alternative medicines for AIDS in resource-poor settings: insights from exploratory anthropological studies in Asia and Africa. J Ethnobiol Ethnomed. 2008 Jul 10;4:16.

37 Kirtane AR, Rothenberger MK, Frieberg A, Nephew K, Schultz-Darken N, Schmidt T, et al. Evaluation of vaginal drug levels and safety of a locally administered glycerol monolaurate cream in rhesus macaques. J Pharm Sci. 2017 Jul;106(7):1821-7..

38 Vandenberghe C, St-Pierre V, Fortier M, Castellano CA, Cuenoud B, Cunnane SC. Medium chain triglycerides modulate the ketogenic effect of a metabolic switch. Front Nutr. 2020 Jan 31;7(3):3.
39 Tay MZ, Poh CM, Rénia L, MacAry PA, Ng LFP. The trinity of COVID-19: immunity, inflammation and intervention. Nat Rev Immunol. 2020 Jun;20(6):363-74.

40 Hannan A, Rahman A, Rahman S, Sohag AAM, Dash R, Hossain KS, et al. Intermittent fasting, a possible priming tool for host defense against SARS-CoV-2 infection: crosstalk among calorie restriction, autophagy and immune response. Immunol Lett. 2020;226: $38-45$.

41 Martínez-Vallespín B, Vahjen W, Zentek J. Effects of medium-chain fatty acids on the structure and immune response of IPEC-J2 cells. Cytotechnology. 2016 Oct;68(5):1925-36.

42 Faris MAIE, Kacimi S, Al-Kurd RA, Fararjeh MA, Bustanji YK, Mohammad MK, et al. Intermittent fasting during Ramadan attenuates proinflammatory cytokines and immune cells in healthy subjects. Nutr Res. 2012 Dec 01; 32(12):947-55.
43 Faris MAIE, Jahrami HA, Obaideen AA, Madkour MI. Impact of diurnal intermittent fasting during Ramadan on inflammatory and oxidative stress markers in healthy people: systematic review and meta-analysis. J Nutr Intermed Metab. 2019 Mar 01;15:1826.

44 Moore JB, June $\mathrm{CH}$. Cytokine release syndrome in severe COVID-19. Science. 2020; 368(6490):473-4.

45 Tanaka T, Narazaki M, Kishimoto T. Immunotherapeutic implications of IL-6 blockade for cytokine storm. Immunotherapy. 2016; 8(8):959-70.

46 Serreli G, Deiana M. Extra virgin olive oil polyphenols: modulation of cellular pathways related to oxidant species and inflammation in aging. Cells. 2020;9(2):478. 